\title{
Occurrence of Dengue Virus Infection with Multiple Serotypes in Central Karnataka, India
}

\author{
VinodKumar C. Shivaswamy. ${ }^{1}$ Prasad B. Shivanagowda ${ }^{2}$ Kalappanavar N. Kenchappa ${ }^{2}$ \\ Jayasimha V. Lakshminarayan ${ }^{1}$ \\ ${ }^{1}$ Department of Microbiology, S.S. Institute of Medical Sciences and \\ Research Centre, Davanagere, Karnataka, India \\ 2 Department of Pediatrics, S.S. Institute of Medical Sciences and \\ Research Centre, Davanagere, Karnataka, India \\ Address for correspondence VinodKumar Chickmagalur Shivaswamy, \\ $\mathrm{PhD}$ (Microbiology), PhD (Medical Microbiology), Department of \\ Microbiology, S.S. Institute of Medical Sciences and Research Centre, \\ $\mathrm{NH}-4$, Davanagere 577003, Karnataka, India \\ (e-mail: vinodmicro@gmail.com).
}

J Lab Physicians 2022;14:37-42.

\begin{abstract}
Keywords

- dengue

- dengue-like illness

- concurrent infection

- RT-PCR

- NS1 antigen

Objective To find the incidence of concurrent infection of dengue and correlate the difference in clinical features, laboratory diagnoses, and outcomes between dengue and dengue-like illnesses.

Methodology A total of 2,256 patients with suspected dengue fever during the period of July 2014 to June 2020 as per the WHO case definition for dengue fever were enrolled in the study. All patients admitted with a suspected dengue fever were studied in detail in terms of demographic features, clinical features, and laboratory profiles. Two blood samples were collected from each patient with a history of fever for 5 to 7 days. Investigation consisted of CBC, Widal test, malarial card test, Weil Felix test, Scrub typhus test, chikungunya, dengue parameters such as dengue NS1, IgM, and IgG, and real-time PCR for dengue serotypes were performed for each sample.

Results A total of 1,412 males (62.6\%) and 844 females (37.4\%) of age 2 to 44 years were hospitalized. Out of 2,256 clinically suspected dengue cases, 1,306 cases were positive and 950 were negative by RT-PCR test. Fever was the most common clinical features among the RT-PCR-positive cases, followed by retroorbital pain (85.9\%), flushing in $77.5 \%$, and rashes in $84.8 \%$ of patients. ARDS was seen in $9.7 \%$ and splenomegaly in $27.5 \%$ patients. A platelet count of less than 100,000 was observed in 1,838 (81.5\%) patients, and a platelet count of less than 20,000 was observed in 147 (6.5\%) patients.

Of 2,256 samples, 1,306 (57.9\%) tested positive for dengue viral RNA by RT-PCR. Also, 798 cases were infected with a single DENV serotype, and 608 had a concurrent infection. Of the 798 single DENV serotype infection cases, 392 (54.2\%) were typed as DENV-2 and 218 (29.2\%) as DENV-3. Coinfection with serotypes DENV-2 and DENV-3 was found to account for $67.8 \%$ of all concurrent infections.

Conclusion The study showed that dengue fever with concurrent infection with multiple serotypes is on the rise, and an occurrence of recombination may lead to the emergence of more virulent strains showing varied clinical presentations.
\end{abstract}

published online November 23, 2021
DOI https://doi.org/

10.1055/s-0041-1739536. ISSN 0974-2727.
(C) 2021. The Indian Association of Laboratory Physicians. All rights reserved.

This is an open access article published by Thieme under the terms of the Creative Commons Attribution-NonDerivative-NonCommercial-License, permitting copying and reproduction so long as the original work is given appropriate credit. Contents may not be used for commercial purposes, or adapted, remixed, transformed or built upon. (https://creativecommons.org/ licenses/by-nc-nd/4.0/)

Thieme Medical and Scientific Publishers Pvt. Ltd., A-12, 2nd Floor, Sector 2, Noida-201301 UP, India 


\section{Introduction}

Dengue virus infections are a significant cause of morbidity and mortality and lead to adverse economic effects in many developing tropical countries. ${ }^{1}$ The incidence of dengue fever is on the rise worldwide, and in some areas of Asia, complications of the disease are a leading cause of serious illness and death in children.,3 Over the past two decades in India, there has been a dramatic increase in dengue fever (DF), dengue hemorrhagic fever (DHF), and dengue shock syndrome (DSS), and their epidemics. ${ }^{4-6}$ The identification of dengue cases is by distinct clinical features, but they can present with varied manifestations. ${ }^{7-9}$ Dengue remains a puzzling disease in many aspects, such as the virus-vector and host-virus relationship, and clinical expression variability. ${ }^{10-12}$ The dengue epidemics in India are cyclical and are more frequent, expanding into the rural areas and dengue viral strains are circulating in the community either by local evolution or importations, leading to a heterogeneous distribution of serotypes/genotypes in different geographical areas. ${ }^{10,11}$ This in turn alters the infectivity of the virus and gets reflected as changes in the clinical spectrum of the disease. The aim of the present study was to find the incidence of concurrent infection of dengue virus and to correlate the difference in clinical features, laboratory diagnoses, and outcomes between dengue and dengue-like illness

\section{Materials and Methods}

\section{Ethical Clearance}

The protocol was approved by the Institutional Ethics Review Board. Consent was obtained from all patients.

\section{Type of the Study}

Prospective study.

Study Duration: Six years (July 2014-June 2020).

\section{Inclusion Criteria}

According to specific inclusion criteria, 2,256 patients with fever (presenting within 5-7 days of onset with body temperature above $100^{\circ} \mathrm{F}$ at the time of blood sample collection) and fulfilling the case definition criteria of DF and DHF of the World Health Organization (WHO) were included in the study. Clinical and demographic data were collected by interviewing the patients or their attendants and meticulous physical examination of the patients conducted by their treating physician.

\section{Specimen Collection}

Two blood samples were collected from each patient and one blood sample was sent on ice to the molecular laboratory for the detection of dengue viruses, the NS1 antigen, and $\operatorname{IgM}$ and $\operatorname{IgG}$ antibodies. The second sample was used for a complete blood hemogram and for other investigations such as Widal test, malarial test by card and peripheral smear, chikungunya test by card method and for typhus fever test. The clinical basis for diagnosing patients with dengue virus infection was based on the WHO definitions. ${ }^{11}$

Reports of hematological investigations, dengue serology, and data obtained from daily follow-up were analyzed. Hospitalized patients were categorized into DF, DHF, and DSS according to the WHO severity grading scale. ${ }^{11}$ The blood indices were initially measured on a continuous scale and then categorized on the basis of clinically meaningful cut offs. Thrombocytopenia was defined as a platelet count less than 100,000 cells $/ \mathrm{mm}^{3}$ blood. A hemotocrit more than $20 \%$ rise was considered raised. Similarly, leucopenia was defined as a white cell count less than 4,000 cells $/ \mathrm{mm}^{3}$.

Viral RNA was extracted from serum samples using the QIAamp Viral RNA mini kit (Qiagen, Germany) according to the manufacturers' instructions. The extracted RNA was stored at $-70^{\circ} \mathrm{C}$ or immediately used for RT-PCR. RT and PCR were performed in one tube using a universal primer and a one-step RT kit (QIAGEN, GmbH, Hilden, Germany); the reaction was then placed in a thermal cycler (Eppendorf, Germany). The preliminary product was further used for nested PCR in another reaction tube. ${ }^{12}$ Nested PCR was performed with a thermal cycler. The secondary PCR product was subjected to agarose gel electrophoresis using a $2 \%$ agarose gel (Bangalore Gene) in Tris-borate buffer, followed by staining with ethidium bromide and visualization on a UV transilluminator at $302 \mathrm{~nm}$. The NS1 antigen and IgM and IgG antibodies were detected with immuno-chromatographic test (ICT). The test kit used was the dengue NS1 antigen and antibody combi card supplied by J. Mitra and Co. Pvt. Ltd. (New Delhi, India). ${ }^{13}$

\section{Statistical Analysis}

Data were analyzed using the Epi-info software. The categorical data were shown in terms of numbers and percentages and analyzed using the Z-test for proportions and chisquare test.

\section{Results}

Out of 2,256 patients enrolled for the study, 1,412 (62.6\%) were male and $844(37.4 \%)$ were female. In addition, 1,356 (60.1\%) were from rural area and 900 (39.9\%) were urban population. The age distribution is shown in - Table 1 . The study population age ranged from 2 to 44 years.

Out of 2,256 clinically suspected dengue cases, 1,306 were positive and 950 were negative by RT-PCR test.

Various clinical features are summarized in -Table 2. Among the RT-PCR-positive dengue cases, fever was the most common clinical presentation occurring in all patients. There was no specific pattern of fever, and the fever ranged from $38^{\circ} \mathrm{C}$ to $40^{\circ} \mathrm{C}$. Other common clinical features were retroorbital pain (85.9\%), flushing in $77.5 \%$, and rashes in $84.8 \%$ of patients. ARDS was seen in $9.7 \%$, splenomegaly in $27.5 \%$, ascites in $20.3 \%$, and encephalopathy in $3.4 \%$ of patients.

Among the RT-PCR-negative dengue cases, fever was the most common clinical presentation occurring in all patients. Retroorbital pain was seen in $91.6 \%$ of patients, $74.9 \%$ of patients exhibited flushing, rashes were seen in $34.9 \%$ of 
Table 1 Demographic, sex, and age of study subjects

\begin{tabular}{|l|l|l|l|l|}
\hline \multicolumn{2}{|l|}{ Features } & \multicolumn{2}{l}{ RT-PCR } & \multirow{2}{*}{ Total } \\
\cline { 3 - 4 } \multicolumn{2}{|c|}{} & Positive & Negative & \\
\hline \multirow{3}{*}{ Demographic } & Rural & 840 & 516 & 1,356 \\
\cline { 2 - 4 } & Urban & 466 & 434 & 900 \\
\hline \multirow{3}{*}{ Sex } & Male & 789 & 623 & 1,412 \\
\cline { 2 - 5 } & Female & 517 & 327 & 844 \\
\hline \multirow{5}{*}{ Age } & $<5$ y & 129 & 149 & 278 \\
\cline { 2 - 5 } & $6-10$ y & 522 & 304 & 826 \\
\cline { 2 - 5 } & $11-15$ y & 379 & 263 & 642 \\
\cline { 2 - 4 } & $>15$ y & 286 & 224 & 510 \\
\hline
\end{tabular}

patients, ARDS was seen in $19.8 \%$, ascites in $5.7 \%$, and encephalopathy in $9.7 \%$ of patients (- Table $\mathbf{2}$ ).

A platelet count less than 100,000 was observed in 1,838 (81.5\%) patients, a platelet count of 50,000 to 100,000 was observed in 835 (37\%) patients, a platelet count of 20,000 to 50,000 was observed in 856 (37.9\%) patients, and a platelet count less than 20,000 was observed in 147 (6.5\%) patients (-Table 3).

Of 2,256 samples, 1,306 (57.9\%) tested positive for dengue viral RNA by RT-PCR. Seven hundred ninety-eight cases were infected with a single DENV serotype, and 608 had a concurrent infection with two DENV serotypes. Of the 798 singleinfection cases, 392 (54.2\%) were typed as DENV-2, 218 (29.2\%) as DENV-3, 114 (8.3\%) as DENV-1, and 74 (8.3\%) as DENV-4. DENV-2 dominated the outbreak, accounting for $49.1 \%$ of the positive samples, followed by DENV-3 (27.3\%).

The overall prevalence of concurrent infections was $46.6 \%$. Coinfection with serotypes DENV-2 and DENV-3 was found to account for $67.8 \%$ of all concurrent infections. Other combinations included the following: DENV-1 and DENV-3 (81 of $608,16.7 \%$ ); DENV-2 and DENV-4 (48 of $608,11.1 \%$ ); DENV-2 and DENV-4 (48 of 608, 5.6\%); and DENV-3 and DENV-4 (44 of $608,5.6 \%$ ). Thus, DENV-2 and DENV-3 were the most commonly combined serotypes observed over the period.

Table 2 Clinical manifestations of patients with RT-PCR-positive and -negative cases

\begin{tabular}{|c|c|c|c|c|}
\hline \multirow[t]{3}{*}{ Clinical features } & \multicolumn{4}{|l|}{ RT-PCR } \\
\hline & \multicolumn{2}{|l|}{ Positive } & \multicolumn{2}{|l|}{ Negative } \\
\hline & Number & Percentage & Number & Percentage \\
\hline Fever & 1,306 & 100.0 & 950 & 100.0 \\
\hline Retro orbital pain & 1,122 & 85.9 & 870 & 91.6 \\
\hline Flushing & 1,012 & 77.5 & 712 & 74.9 \\
\hline Rash & 1,108 & 84.8 & 332 & 34.9 \\
\hline ARDS & 127 & 9.7 & 188 & 19.8 \\
\hline Encephalopathy & 44 & 3.4 & 92 & 9.7 \\
\hline Hepatitis & 141 & 10.8 & 57 & 6.0 \\
\hline Malena & 73 & 5.6 & 361 & 38.0 \\
\hline Epistaxis & 12 & 0.9 & 18 & 1.9 \\
\hline Hematemesis & 13 & 1.0 & 6 & 0.6 \\
\hline Hepatomegaly & 99 & 7.6 & 327 & 34.4 \\
\hline Splenomegaly & 38 & 2.9 & 104 & 10.9 \\
\hline Ascites & 265 & 20.3 & 54 & 5.7 \\
\hline Pleural effusion & 164 & 12.6 & 39 & 4.1 \\
\hline Cyanosis & 14 & 1.1 & 8 & 0.8 \\
\hline Convulsion & 55 & 4.2 & 113 & 11.9 \\
\hline Oliguria & 14 & 1.1 & 10 & 1.1 \\
\hline Hypoglycemia & 16 & 1.2 & 6 & 0.6 \\
\hline Abscess & 14 & 1.1 & 21 & 2.2 \\
\hline Pneumonia & 17 & 1.3 & 32 & 3.4 \\
\hline Hematuria & 8 & 0.6 & 6 & 0.6 \\
\hline Gum bleeding & 2 & 0.2 & 6 & 0.6 \\
\hline Sub conjunctival hemorrhage & 2 & 0.2 & 22 & 2.3 \\
\hline Total & 1,306 & 100.0 & 950 & 100.0 \\
\hline
\end{tabular}


Table 3 Platelet count during the admission among patients with suspected dengue fever

\begin{tabular}{|c|c|c|c|c|c|}
\hline \multirow[t]{2}{*}{ Age (Years) } & \multicolumn{4}{|c|}{ Platelet count (cells $/ \mathrm{mm}^{3}$ ) } & \multirow[t]{2}{*}{ Total } \\
\hline & $<20,000$ & 20,001 to 50,000 & 50,001 to 100,000 & $>100,000$ & \\
\hline$<5$ & 20 & 120 & 80 & 58 & 278 \\
\hline $6-10$ & 78 & 328 & 294 & 126 & 826 \\
\hline $11-15$ & 42 & 232 & 318 & 50 & 642 \\
\hline$>15$ & 7 & 175 & 143 & 180 & 510 \\
\hline Total & 147 & 856 & 835 & 414 & 2,256 \\
\hline
\end{tabular}

Out of 1,306 RT-PCR-positive cases, 1,148 were found to have positive serology (NS1, NS1 and IgM, IgM,) and 86 cases were positive among 921 dengue RT-PCR-negative cases. Among the serologically positive cases, 1,134 cases were NS1 positive alone, 122 cases were NS1 and IgM positive, and 12 cases were only IgM positive among the 1,306 cases of RTPCR-positive cases (-Table 4). Among RT-PCR-negative cases, 15 cases were NS1-positive, 22 were NS1 and IgM positive, 8 cases were IgM alone positive, 12 were IgM and IgG positive, and 29 cases were positive for IgG in RT-PCRnegative cases ( - Table 4 ).

Among the 1,306 RT-PCR-positive dengue cases, 660 (50.5\%), $376(28.8 \%)$, and $270(20.7 \%)$ were classified as classical DF, as well as DHF, and DSS.

Out of 950 RT-PCR-negative cases, 44 samples tested positive for malaria by card test and also by peripheral smear, 69 samples were positive for enteric fever by Widal test, and 126 samples were positive for scrub typhus by ELISA test, and 10 were positive for chikungunya.

\section{Discussion}

Dengue virus causes a broad spectrum of illnesses, ranging from mild undifferentiated fever to classical DF, as well as
DHF and DSS. ${ }^{3,4,14}$ Dengue is a major public health problem in central Karnataka. Over the past 15 years, we have been observing varied clinical manifestations of dengue, ${ }^{4,7,12}$ which are rather different from the past reports from this region as well as from other parts of the country.

The seasonal drift of dengue virus infection is revealed by the zenith of positive cases observed during the month of August to December, which is in concordance with previous outbreaks. ${ }^{3,7,14-16}$ The majority of patients $(62.6 \%)$ in our series were males and from rural area $(60.1 \%)$. The commonest age group involved were young children varying from 6 to 10 years of age. In the present study, 2,256 patients were admitted with suspected DF. Among these, 1,306 patients (57.8\%) were confirmed to have the dengue disease by RTPCR, and 44 samples tested positive for malaria by card test and also by peripheral smear, 69 samples were positive for enteric fever by Widal test, and 126 samples were positive for scrub typhus by ELISA test, and 10 were positive for chikungunya.

Most developing countries have epidemics of febrile illnesses, which can be confused with DF. ${ }^{14,16}$ A recent review of published studies was unable to make any conclusions on the signs and symptoms that can clinically distinguish dengue from other febrile illnesses. ${ }^{7,9,14}$

Table 4 Comparison of dengue serology with RT-PCR-positive cases

\begin{tabular}{|c|c|c|c|c|c|}
\hline \multirow[t]{3}{*}{ Dengue serology } & \multirow[t]{3}{*}{ Result } & \multicolumn{4}{|l|}{ RT-PCT } \\
\hline & & \multicolumn{2}{|l|}{ Positive } & \multicolumn{2}{|l|}{ Negative } \\
\hline & & Number & Percentage & Number & Percentage \\
\hline \multirow[t]{2}{*}{ NS1 } & Positive & 1,134 & 86.8 & 15 & 1.6 \\
\hline & Negative & 172 & 13.2 & 935 & 98.4 \\
\hline \multirow[t]{2}{*}{$\mathrm{NS1}+\operatorname{lgM}$} & Positive & 122 & 9.3 & 22 & 2.3 \\
\hline & Negative & 1,184 & 91.7 & 928 & 97.7 \\
\hline \multirow[t]{2}{*}{$\lg M$} & Positive & 12 & 0.9 & 8 & 0.8 \\
\hline & Negative & 1,294 & 99.1 & 942 & 99.2 \\
\hline \multirow[t]{2}{*}{$\lg M+\lg G$} & Positive & 0 & 0 & 12 & 1.3 \\
\hline & Negative & 1,306 & 100 & 938 & 98.7 \\
\hline \multirow[t]{2}{*}{$\lg G$} & Positive & 0 & 0 & 29 & 3.1 \\
\hline & Negative & 1,306 & 100 & 921 & 96.9 \\
\hline
\end{tabular}


Even in the present study, there was not much significant difference in the clinical features of DF (RT-PCR-positive) and dengue-like illness (RT PCR-negative). Dengue fever is generally described as a short febrile illness. The WHO criteria mention an illness of 2 to 7 days' duration. ${ }^{5}$ In the present study, we have observed a longer duration of fever than in previous years. The mean duration of fever in survivors in this study was almost 7.4 days and the longest duration of fever was more than 3 weeks 4 days in 32 patients. The predominant clinical features were fever, retroorbital pain, flushing, and myalgia, and vomiting was the most frequent symptom, which has also been observed in other studies. ${ }^{5-7}$ The major difference from the previous report is the frequent occurrence of acute respiratory distress syndrome, splenomegaly, prolonged fever, and encephalopathy. In addition, 9.7\% of DF cases showed ARDS, while $19.8 \%$ of dengue-like cases showed ARDS.

Although hepatomegaly is among the WHO clinical criteria for DF, splenomegaly is not generally held to be a feature of dengue infection. Earlier studies in India do not describe a high frequency of splenomegaly. ${ }^{7-12}$ But in our study, we observed $27.5 \%$ of the cases had splenomegaly among DF. Our study corroborates with a recent study from Delhi that has reported a higher percentage $(32.4 \%)$ of splenomegaly in children with dengue. ${ }^{7}$

Even though a high incidence of encephalopathy has been reported in several studies, it was observed in $3.4 \%$ of cases in DF and 9.7\% in dengue-like fever in our study.

On an analysis of laboratory findings, it was observed that platelets were below $100,000 / \mathrm{mm}^{3}$ in $81.5 \%$ of dengue suspected cases and $6.5 \%$ of cases had platelets less than $20,000 / \mathrm{mm}^{3}$ Thrombocytopenia is thought to be due to depression of the bone marrow observed in acute stage of dengue virus infection. Other explanations are direct infection of megakaryocytes by the virus, leading to increased destruction of platelets or the presence of antibodies directed against platelets. ${ }^{17}$ A leukocyte count less than $4,000 / \mathrm{mm}^{3}$ was seen in $77.6 \%$ of RT-PCR-positive dengue cases and hematocrite value more than $20 \%$ for age and sex was observed in $65 \%$ of cases. Coagulopathy is also frequent in most patients with DSS. In our study, prolongation of PTT was quite common in DSS cases of both groups. The other important abnormality was raised liver enzymes. sGOT and sGPT were raised above the normal limit in $47.7 \%$ of cases and $46.2 \%$ of cases, respectively, in the study cohort.

The most challenging problem associated with patient management in dengue infection is rapid diagnosis. Although the commercially available MAC ELISAs or ICT offer improvements over other conventional assays for the diagnosis, they do not offer serotype-specific diagnosis. Diagnosis based on the detection of antigen (NS1) can be achieved on first day, but in the present study, the NS1 was positive only in $96.2 \%$ of cases and detection of IgM antibodies can only be achieved after 5 to 7 days of illness. The detection of concurrent infections can be made by virus isolation in tissue culture, followed by indirect immunofluorescence using serotype-specific monoclonal antibodies and/or RT-PCR. ${ }^{11}$ However, RT-PCR offers accuracy and speed along with serotype-specific diagnosis of various circulating dengue viruses and information about co-circulation of different subtypes. It is now notably clear that the epidemics caused by multiple dengue virus serotypes have become more frequent on a global basis in the last 18 years. ${ }^{9}$ General belief is that concurrent infections by different dengue serotypes occur during epidemics only, where multiple virus serotypes are being transmitted. The co-circulation of multiple dengue serotypes in the same region has been documented in several countries for decades. ${ }^{14-16}$ It has been hypothesized that concurrent infections by multiple dengue virus serotypes may influence the clinical expression of the disease. This is considered as a single major factor for the emergence of DHF.

The detection of dengue virus RNA by RT-PCR showed that DENV-2 and DENV-3 were the most common etiologic agents, followed by DENV-1 in this period of study. Only three dengue virus serotypes were found to be co-circulating. Previously from Delhi and its surrounding areas, only two concurrent dengue cases were identified ${ }^{18}$ but now the number has significantly increased. One fact that may be of considerable importance in concurrent infections is the occurrence of recombination events. Their recombination may lead to the emergence of more virulent strains.

\section{Conflict of Interest}

None declared.

\section{Acknowledgments}

The authors would like to thank Vision Group of Science and Technology for the grants and the host institute for the facilities.

\section{References}

1 Garg A, Garg J, Rao YK, Upadhyay GC, Sakhuja S. Prevalence of dengue among clinically suspected febrile episodes at a teaching hospital in North India J. Infect. Dis. Immun. 2011;3(05):85-89

2 Martina BE, Koraka P, Osterhaus AD. Dengue virus pathogenesis: an integrated view. Clin Microbiol Rev 2009;22(04):564-581

3 Siddiqui TS, Rehman AU, Ahmed B. Etiology of strokes and hemiplegia in children presenting at Ayub Teaching Hospital, Abbottabad. J Ayub Med Coll Abbottabad 2006;18(02):60-63

4 Ratageri VH, Shepur TA, Wari PK, Chavan SC, Mujahid IB, Yergolkar PN. Clinical profile and outcome of dengue fever cases. Indian J Pediatr 2005;72(08):705-706

5 Kumar R, Tripathi S, Tambe JJ, Arora V, Srivastava A, Nag VL. Dengue encephalopathy in children in Northern India: clinical features and comparison with non dengue. J Neurol Sci 2008;269 (1-2):41-48

6 Kumar R, Tripathi P, Tripathi S, Kanodia A, Venkatesh V. Prevalence of dengue infection in north Indian children with acute hepatic failure. Ann Hepatol 2008;7(01):59-62

7 Kumar A, Rao CR, Pandit V, Shetty S, Bammigatti C, Samarasinghe CM. Clinical manifestations and trend of dengue cases admitted in a tertiary care hospital, udupi district, karnataka. Indian J Community Med 2010;35(03):386-390

8 Kabra SK, Jain Y, Singhal T, Ratageri VH. Dengue hemorrhagic fever: clinical manifestations and management. Indian J Pediatr 1999;66(01):93-101

9 Gunasekaran P, Kaveri K, Mohana S, et al. Dengue disease status in Chennai (2006-2008): a retrospective analysis. Indian J Med Res 2011;133:322-325 
42 Dengue with Multiple Serotypes in Central Karnataka Shivaswamy et al.

10 Holmes EC, Burch SS. The causes and consequences of genetic variation in dengue virus. Trends Microbiol 2000;8(02):74-77

11 Rico-Hesse R, Harrison LM, Salas RA, et al. Origins of dengue type 2 viruses associated with increased pathogenicity in the Americas. Virology 1997;230(02):244-251

12 Vaughn DW, Green S, Kalayanarooj S, et al. Dengue viremia titer, antibody response pattern, and virus serotype correlate with disease severity. J Infect Dis 2000;181(01):2-9

13 Chaterji S, Allen JC Jr, Chow A, Leo YS, Ooi EE. Evaluation of the NS1 rapid test and the WHO dengue classification schemes for use as bedside diagnosis of acute dengue fever in adults. Am J Trop Med Hyg 2011;84(02):224-228

14 Yamada K, Nawa M, Takasaki T, Yabe S, Kurane I. Laboratory diagnosis of dengue virus infection by reverse transcriptase polymerase chain reaction (RT-PCR) and IgM-capture enzymelinked immunosorbent assay (ELISA).Jpn J Infect Dis 1999;52(04): 150-155

15 Wilder-Smith A, Earnest A, Paton NI. Use of simple laboratory features to distinguish the early stage of severe acute respiratory syndrome from dengue fever. Clin Infect Dis 2004;39(12): $1818-1823$

16 Sabin AB. Research on dengue during World War II. Am J Trop Med Hyg 1952;1(01):30-50

17 Schexneider KI, Reedy EA. Thrombocytopenia in dengue fever. Curr Hematol Rep 2005;4(02):145-148

18 Tazeen A, Afreen N, Abdullah M, et al. Occurrence of co-infection with dengue viruses during 2014 in New Delhi, India. Epidemiol Infect 2017;145(01):67-77 